\title{
Leptospirosis as Frequent Cause of Acute Febrile Illness in Southern Sri Lanka
}

\author{
Megan E. Reller, Champika Bodinayake, Ajith Nagahawatte, Vasantha Devasiri, \\ Wasantha Kodikara-Arachichi, John J. Strouse, Judith E. Flom, J. Stephen Dumler, \\ and Christopher W. Woods
}

To determine the proportion of fevers caused by leptospirosis, we obtained serum specimens and epidemiologic and clinical data from patients in Galle, Sri Lanka, March-October 2007. Immunoglobulin M ELISA was performed on paired serum specimens to diagnose acute (seroconversion or 4-fold titer rise) or past (titer without rise) leptospirosis and seroprevalence (acute). We compared (individually) the diagnostic yield of acute-phase specimens and clinical impression with paired specimens for acute leptospirosis. Of 889 patients with paired specimens, 120 had acute leptosoirosis and 241 had past leptospirosis. The sensitivity and specificity of acute-phase serum specimens were $17.5 \%$ (95\% confidence interval [Cl] 11.2\%-25.5\%) and $69.2 \%(95 \% \mathrm{Cl} 65.5 \%-72.7 \%)$, respectively, and of clinical impression $22.9 \%(95 \% \mathrm{Cl} 15.4 \%-32.0 \%)$ and $91.7 \%$ (95\% Cl 89.2\%-93.8\%), respectively. For identifying acute leptospirosis, clinical impression is insensitive, and immunoglobulin $\mathrm{M}$ results are more insensitive and costly. Rapid, pathogen-based tests for early diagnosis are needed.

$\mathrm{L}$ eptospirosis is an endemic zoonosis in the tropics, where a favorable climate enables the pathogenic spirochete Leptospira interrogans to survive in the environment (1). Furthermore, many tropical residents have repeated direct and indirect exposures to infected animals that excrete leptospires in their urine (2).

Sri Lanka, with a rapidly growing population of $\approx 20$ million, has a reported annual incidence of leptospirosis

Author affiliations: Johns Hopkins University School of Medicine, Baltimore, Maryland, USA (M.E. Reller, J.J. Strouse, J.S. Dumler); University of Ruhuna, Galle, Sri Lanka (C. Bodinayake, A. Nagahawatte, V. Devasiri, W. Kodikara-Arachichi); Johns Hopkins School of Public Health, Baltimore (J.E. Flom); and Duke University School of Medicine, Durham, North Carolina, USA (C.W. Woods)

DOI: $10.3201 /$ eid1709.100915 of 5.4 cases $/ 100,000$ persons, the sixth highest incidence worldwide (3). Approximately $28 \%$ of Sri Lanka's workforce is employed in agriculture, and reported cases of leptospirosis fluctuate with rainfall and farming cycles. Historically, $\approx 200$ cases per million population per year have been reported from the southern and north-central regions, where the disease is hyperendemic (3). However, incidence rates are imprecise estimates because leptospirosis is easily confused with undifferentiated fever of other causes (1), and few cases are laboratory confirmed (4). In the past 2 decades, clinical cases have been increasingly reported (5), including $>7,000$ cases in 2008 (6).

To determine the prevalence of acute and past leptospirosis in southern Sri Lanka, assess tools for acute diagnosis, and identify associated features, we collected epidemiologic and clinical data and paired serum specimens from a prospective cohort of children and adults with undifferentiated fever. The institutional review boards of the University of Ruhuna, Johns Hopkins University, and Duke University Medical Center approved this study.

\section{Materials and Methods}

\section{Study Participants}

We recruited patients in the emergency department, acute care clinics, and adult and pediatric wards of the Karapitiya Teaching Hospital in Galle, the largest (1,300bed) hospital in southern Sri Lanka, during March-October 2007. We enrolled consecutive febrile $\left(38^{\circ} \mathrm{C}\right.$, tympanic) patients $\geq 2$ years old without antecedent ( $\leq 7$ days) trauma or hospitalization who sought treatment during clinic hours (8:00 AM-4:00 PM Monday-Friday, and 8:00 AM-2:00 PM Saturday). Study doctors verified patient eligibility and willingness to return for follow-up and obtained written informed consent from patients ( $\geq 18$ years of age) or 
parents (of those $<18$ years of age) and assent from those $12-17$ years of age.

Study personnel recorded structured epidemiologic and clinical data, including duration of illness and the clinical provider's presumptive diagnosis, on a standardized form. Study doctors then obtained blood for on-site clinician-requested testing and subsequent off-site research-related testing. Patients returned for clinical and serologic follow-up 2-4 weeks later or were visited at home if they were unable to return and could be located. Blood was centrifuged, and serum specimens were frozen on site at $-80^{\circ} \mathrm{C}$, shipped on dry ice, and thawed only when separated into aliquots and when tested.

\section{Serologic Testing}

We tested paired serum specimens for the presence of specific Leptospira immunoglobulin (Ig) M by ELISA (Institut Viron Serion GmgH, Warburg, Germany), according to the manufacturer's instructions. Briefly, rheumatoid factor (RF)-absorbent was first diluted 1:4 in buffer. Serum specimens from patients and controls were then diluted (1:100) in RF-absorbent buffer to accomplish removal of IgM RF, transferred to antigen-coated microtest wells, and incubated at $37^{\circ} \mathrm{C}$ for $60 \mathrm{~min}$. After wells were washed with phosphate-buffered saline, antihuman IgM (conjugated to alkaline phosphatase and $p$-nitrophenylphosphate) was added. After incubation of the wells for $20 \mathrm{~min}$, sodium hydroxide was added to each well to stop the reaction, and the absorbance at $405 \mathrm{~nm}$ was measured.

The ELISA provided qualitative results-positive, negative, and equivocal (borderline positive/negative). Using a standard curve and evaluation table provided with the kit, we obtained the optical density measurements, which were adjusted for plate-to-plate variation with a correction factor and gave quantitative results that correlated with titers (7).

\section{Case Definitions}

Acute leptospirosis was defined as definitive seroconversion (negative acute-phase serum specimen to positive convalescent-phase serum specimen) or the equivalent of a 4-fold rise in IgM titer. We excluded from analyses of acute leptospirosis specimens with equivocal IgM test results or those lacking a convalescent-phase sample.

Past leptospirosis was defined as stable or decreasing IgM titers. We excluded from analyses of past leptospirosis specimens with equivocal IgM test results or those lacking a convalescent-phase sample. IgM seroprevalence was defined as the prevalence of leptospirosis by Leptospira IgM in acute-phase serum specimens, independent of whether a convalescent-phase specimen was obtained or its result.

\section{Statistical Analysis}

Proportions were compared by the $\chi^{2}$ test or Fisher exact test and continuous variables by Student $t$ test or the rank sum test if distribution was not normal. Confidence intervals (CIs) for risk ratios were calculated by exact methods. We assessed $\operatorname{IgM}$ in the acute-phase sample for seroprevalence and clinical impression was compared with results of paired-serum specimen testing for acute leptospirosis. We specifically correlated epidemiologic features, duration of illness, and symptoms and signs with serologic test results. Analyses were performed with Stata IC 11.0 (StataCorp LP, College Station, TX, USA).

\section{Results}

\section{Patient Characteristics}

Paired serum specimens were available from 889 $(82.4 \%)$ of 1,079 patients consecutively enrolled. Among those, a diagnosis of acute leptospirosis could be confirmed or refuted for $773(87.0 \%)$ of 889 , because serologic results were inconclusive for 116 . The likelihood of a participant's returning for convalescent-phase serum sampling and clinical follow-up did not differ by age $(p=0.10)$. Female patients were slightly more likely to return for follow-up ( 85.8 vs. $80.6 \% ; p=0.03)$. Most $(90.2 \%)$ patients lived in rural areas and were more likely to return for follow-up than were those who lived in urban areas (83.5 vs. $71.4 \%$; $\mathrm{p}=0.002)$. The proportion with secondary education was similar in the 2 groups ( 21.7 vs. $19.6 \% ; p=0.51)$, as was reported duration of fever and of illness $(\mathrm{p}=0.15$ and $\mathrm{p}=$ 0.13 , respectively).

Of the 773 patients with conclusive serologic results, the median age was 30.1 years (interquartile range [IQR] 19-47 years). More patients were male $(60.6 \%)$ than female, and the median age did not differ by $\operatorname{sex}(\mathrm{p}=0.78)$. The median reported duration of fever and of illness was 3 days (IQR 2-5 days and 2-7 days, respectively). Many $(37.6 \%)$ reported taking an antimicrobial drug before seeking treatment. The median interval between acutephase and convalescent-phase follow-up was 21 days (IQR 15-33 days).

\section{Diagnosis of Acute Leptospirosis}

Acute leptospirosis was confirmed for 120 patients (Figure 1): by seroconversion for 96 patients and by a 4 -fold rise in titer for 24 patients (in 21 acute-phase specimens with positive results and in 3 acute-phase specimens with equivocal results); acute leptospirosis was excluded for 653 patients. Data on presumptive clinical diagnosis were available for 714 patients, including for 109 of 120 with acute leptospirosis. Of these patients, 25 received a correct diagnosis of acute leptospirosis, and 84 received an incorrect diagnosis of another disease. The sensitivity 


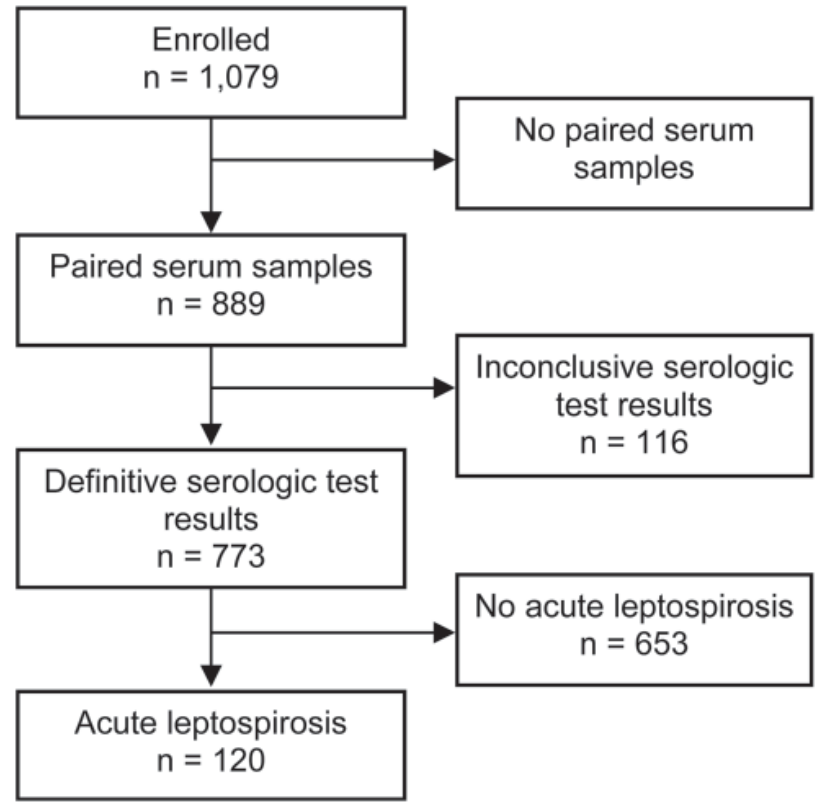

Figure 1. Flowchart indicating selection of study participants with a diagnosis of acute leptospirosis, southern Sri Lanka, 2007.

and specificity of clinical impression were $22.9 \%(95 \%$ CI $15.4 \%-32.0 \%$ ) and $91.7 \%$ (95\% CI $89.2 \%-93.8 \%$ ), respectively. Finally, 279 patients were seropositive at enrollment, including 201 with past leptospirosis, 40 with possible recent leptospirosis (second specimen equivocal), 21 with acute leptospirosis, and 57 without paired serum specimens. Therefore, if acute-phase IgM had been used to diagnose acute leptospirosis instead of paired serum specimens, only 21 of 120 acute infections would have been identified (sensitivity $17.5 \%, 95 \%$ CI $11.2 \%-25.5 \%$ ), and 201 of 653 patients without acute leptospirosis would have been given an erroneous diagnosis (specificity $69.2 \%$, $95 \%$ CI $65.5 \%-72.7 \%$ ). Thus, IgM seropositivity at the acute-phase visit correlated poorly with acute leptospirosis.

\section{Demographic Features of Patients with Acute or Past Leptospirosis}

The demographic characteristics of patients are listed in Table 1. Those with acute or past leptospirosis were older (median 32 years vs. 27 years; $p=0.01$ ) than those without acute or past leptospirosis. Reporting exposure to paddy fields (relative risk [RR] 1.9, 95\% CI 1.6-2.1; $\mathrm{p}<0.0001)$ and working as a farmer (RR $1.9,95 \%$ CI 1.6 $2.3 ; \mathrm{p}=0.0001)$ were strongly associated with acute and past leptospirosis. Reporting no fresh water exposure and boiling drinking water were protective against leptospirosis. Patients enrolled during March-June were less likely to have acute leptospirosis than those enrolled during July-
October (Figure 2). Children $<10$ years of age were much less likely to have acute or past leptospirosis; in contrast, leptospirosis was more common in older adolescents and young adults (Figure 3 ).

The median duration of illness at hospital visit for those with acute leptospirosis diagnosed by seroconversion was 3 days (IQR 2-5 days), and for those with acute leptospirosis diagnosed by a 4 -fold rise in titer, 4 days (IQR $3-5$ days; $\mathrm{p}=0.09)$. The median interval between serum sampling was 22 days (IQR 15-31 days). The follow-up time was slightly longer for those with leptospirosis diagnosed by 4-fold change in titer than for those with diagnosis by seroconversion (median 26 vs. 20 days; $\mathrm{p}=0.08$ ). The median age was 33.6 years (IQR 18.7-45.6 years), and more patients were male $(69.2 \%)$ than female $(\mathrm{p}=0.07)$.

\section{Clinical Features of Acute Leptospirosis}

Clinical features associated with acute leptospirosis are listed in Table 2 . Headache was the most frequent $(\approx 80 \%)$ symptom reported; lethargy, muscle pain, and joint pain were also reported by $>50 \%$ of patients. Lethargy and cough

\begin{tabular}{|c|c|c|c|}
\hline $\begin{array}{l}\text { Demographic } \\
\text { characteristic }\end{array}$ & $\begin{array}{c}\% \text { With acute } \\
\text { or past } \\
\text { leptospirosis, } \\
n=361\end{array}$ & $\begin{array}{c}\% \text { With neither } \\
\text { acute nor past } \\
\text { leptospirosis, } \\
n=412\end{array}$ & $p$ value \\
\hline Median age, y (IQR) & $32(20-46)$ & $27(16-47)$ & 0.02 \\
\hline Male sex & 60 & 64 & 0.14 \\
\hline \multicolumn{4}{|l|}{ Residence } \\
\hline Urban & 8 & 9 & 0.88 \\
\hline Rural & 92 & 91 & \\
\hline Type of work & & & $<0.0005$ \\
\hline Home & 27 & 25 & \\
\hline Laborer & 26 & 21 & \\
\hline Farmer & 6 & 1 & \\
\hline Merchant & 2 & 4 & \\
\hline Student & 20 & 25 & \\
\hline Other & $20 \dagger$ & 24 & \\
\hline \multicolumn{4}{|l|}{ Animal exposures } \\
\hline Dog & $57 \dagger$ & 54 & 0.43 \\
\hline Rodent & 27 & 30 & 0.35 \\
\hline Cow & 7 & $4 \dagger$ & 0.13 \\
\hline \multicolumn{4}{|l|}{ Swim/bathe/wade } \\
\hline None & 66 & 82 & $<0.0005$ \\
\hline River & 14 & 11 & \\
\hline Paddy field & 19 & 4 & \\
\hline Other & $2 \ddagger$ & 3 & \\
\hline Water source & & & 0.001 \\
\hline Tap & 31 & 33 & \\
\hline Boiled & 6 & 14 & \\
\hline Well & 63 & 52 & \\
\hline Other & 0.3 & 1 & \\
\hline
\end{tabular}

*IQR, interquartile range.

†Does not add to $100 \%$ due to rounding.

$\ddagger$ Adds to $>100 \%$ due to multiple exposures. 


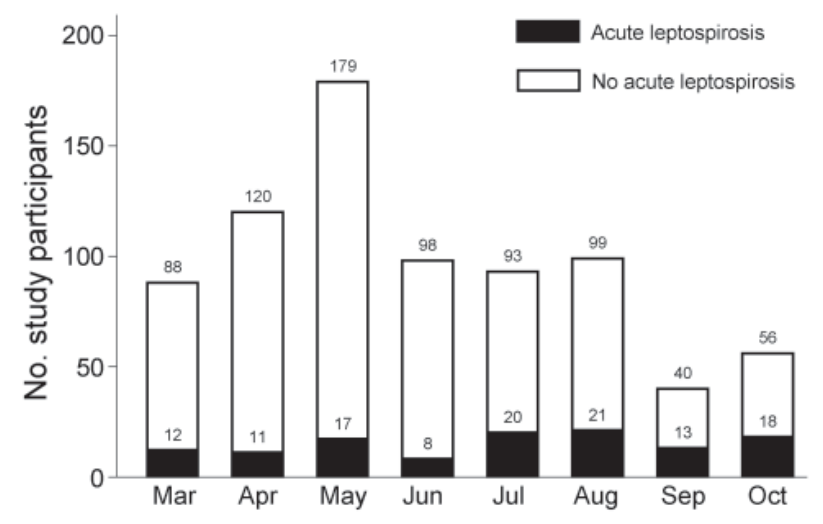

Figure 2. Leptospirosis cases by month among study patients enrolled with fever, southern Sri Lanka, 2007

were reported less often in patients with acute leptospirosis, and oliguria, dysuria, and muscle and joint pain were reported more often. Patients with acute leptospirosis were more likely to have conjunctival suffusion (RR 2.4, 95\% CI 1.7-3.4; $\mathrm{p}<0.0001)$ and less likely to have pharyngeal exudates. Abdominal tenderness and hepatomegaly were slightly more frequent in those with acute leptospirosis. Jaundice, splenomegaly, arthritis, rash, and meningismus were uncommon in both groups. Patients with acute leptospirosis had similar leukocyte counts to patients who did not, and slightly lower hemoglobin concentrations and platelet counts and lower absolute lymphocyte counts. A greater proportion of patients with acute leptospirosis were admitted to the hospital $(84.2 \%$ vs. $70.1 \%)$ than were others with fever $(p=0.002)$, but they did not have a longer stay (median 4 days, IQR 3-6 days; $p=0.83$ ). At the convalescent-phase follow-up visit, patients with acute leptospirosis reported a longer total duration of fever than others (5 days [IQR 3-7 days] vs. 4 days [IQR 3-6 days]; $p=0.008)$. No one with confirmed acute leptospirosis died, but most (11 of 12) deaths occurred before follow-up. Among those who died, the acute-phase serum specimen was IgM-negative for 8 patients, IgM-positive for 2 , and results were equivocal for 1.

\section{Discussion}

We found that leptospirosis was a common, but often clinically unsuspected, cause of fever among unselected patients seeking care in southern Sri Lanka. Farming and rice paddy work were associated with increased risk for leptospirosis, as was exhibiting acute febrile illness during the harvesting season (July-October). In our setting, testing acute-phase serum specimens alone for IgM was less sensitive and specific for diagnosing acute leptospirosis than was diagnosis by observation of clinical features.
Isolation of Leptospira spp. confirms acute infection, but requires special media that must be incubated for up to 13 weeks, and has low sensitivity (1). Therefore, the diagnostic standard for acute leptospirosis is a definitive rise in titer between paired serum specimens (1). Historically, these results have been obtained by the microscopic agglutination test (MAT). Serum specimens are first reacted with live antigen suspensions of different leptospiral serovars. After incubation, the serum-antigen mixtures are examined and titers determined. For paired serum specimens, the highest dilution of serum at which $50 \%$ agglutination occurs must be determined, a laborious and inherently subjective task (1).

Sensitivity is compromised if all locally relevant serovars are not represented, and live cultures of all serovars tested must be maintained whether live or formalin-killed antigens are used. Subculturing many Leptospira spp. weekly is hazardous for personnel, and laboratory-acquired infections occur (1). Reading a MAT requires a dark-field microscope, which is unavailable in most laboratories, including Karapitya Teaching Hospital. Furthermore, a MAT detects both IgM and IgG and lacks sensitivity and specificity when early acute-phase serum specimens alone are tested rather than paired specimens (1). Patients with fulminant illness may die before seroconversion occurs. A MAT may also be less sensitive than an IgM ELISA, even for convalescent-phase specimens. Relative to isolation of Leptospira spp., the reported sensitivities of MATs for acute, late acute-phase, and convalescent-phase serum specimens were $30 \%, 63 \%$, and $76 \%$, respectively, and of IgM ELISA, 52\%, 89\%, and 93\%, respectively, in 1 study in Barbados (8). In another study, results of MAT and IgM ELISA for a single early acute-phase specimen were comparable (49\%) (9).

To overcome the practical pitfalls of MATs, we chose to test paired serum specimens by IgM ELISA, which requires only an inexpensive plate reader, is relatively easy to perform, and provides objective, reproducible results as demonstrated by a parallel comparison of results of

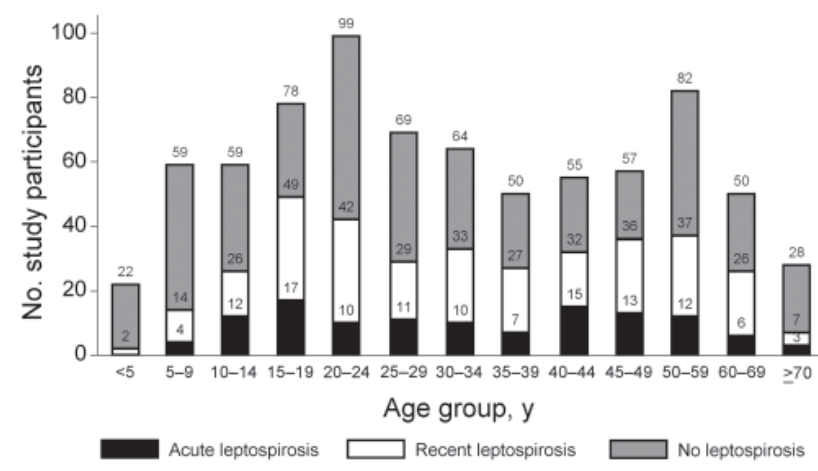

Figure 3. Age distribution of study patients enrolled with fever, southern Sri Lanka, 2007 
Table 2. Clinical characteristics of febrile patients with acute leptospirosis versus those without acute leptospirosis, southern Sri Lanka, $2007^{*}$

\begin{tabular}{|c|c|c|c|}
\hline Clinical characteristic & With acute leptospirosis, $n=120$ & Without acute leptospirosis, $n=653$ & $\mathrm{p}$ value \\
\hline \multicolumn{4}{|l|}{ Symptom } \\
\hline Headache & 81 & 78 & 0.63 \\
\hline Sore throat & 28 & 29 & 0.96 \\
\hline Cough & 44 & 60 & $<0.005$ \\
\hline Dyspnea & 15 & 18 & 0.48 \\
\hline Joint pain & 56 & 43 & $<0.01$ \\
\hline Muscle pain & 62 & 46 & $<0.005$ \\
\hline Lethargy & 58 & 70 & $<0.01$ \\
\hline Abdominal pain & 22 & 18 & 0.41 \\
\hline Emesis & 45 & 37 & 0.10 \\
\hline Diarrhea & 13 & 12 & 0.80 \\
\hline Dysuria & 20 & 13 & $<0.05$ \\
\hline Oliguria & 17 & 8 & $<0.005$ \\
\hline \multicolumn{4}{|l|}{ Sign } \\
\hline Mean temperature, ${ }^{\circ} \mathrm{C}(\mathrm{SD})$ & $38.6(0.6)$ & $38.5(0.6)$ & 0.17 \\
\hline Median heart rate, beats/min (IQR) & $80(72-100)$ & $84(76-96)$ & 0.85 \\
\hline Mean body mass index, $\mathrm{kg} / \mathrm{m}^{2}(\mathrm{SD})$ & $20.7(4.8)$ & $19.6(5.1)$ & $<0.05$ \\
\hline Conjunctival suffusion & 29 & 12 & $<0.0005$ \\
\hline Pharyngeal exudate & 8 & 15 & $<0.05$ \\
\hline Lymphadenopathy & 24 & 23 & 0.73 \\
\hline Jaundice & 2 & 2 & 0.73 \\
\hline Lung crackles & 10 & 14 & 0.27 \\
\hline Tender abdomen & 13 & 9 & 0.21 \\
\hline Hepatomegaly & 8 & 5 & 0.31 \\
\hline \multicolumn{4}{|l|}{ Laboratory parameter, median (IQR) } \\
\hline 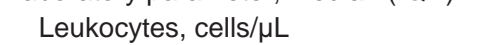 & $7,800(5,700-10,500)$ & $7,900(5,600-11,300)$ & 0.52 \\
\hline Absolute neutrophil count, cells/ $\mu \mathrm{L}$ & $5,530(3,854-8,424)$ & $5,313(3,344-7,952)$ & 0.49 \\
\hline Absolute lymphocyte count, cells/ $\mu \mathrm{L}$ & $1,638(1,210-2,574)$ & $2,140(1,541-2,856)$ & $<0.005$ \\
\hline Hemoglobin, g/dL & $12.3(11.6-13.5)$ & $12.6(11.7-13.8)$ & $<0.05$ \\
\hline Platelets, $\times 1,000 / \mu \mathrm{L}$ & $200(164-256)$ & $231(190-289)$ & $<0.0005$ \\
\hline
\end{tabular}

multiple commercial assays (9). Furthermore, results of performing IgM ELISA on paired serum specimens from patients from various geographic regions have compared favorably (sensitivity $86.5 \%$, specificity $97.0 \%$ ) with MAT results (10). These data suggest that at least $13.5 \%$ of febrile illnesses in our cohort were acute leptospirosis. We assayed paired specimens for IgM instead of IgG, because the kinetics of IgG are more variable $(11,12)$. IgM generally appears within 1 week of symptoms and persists for months to years after infection (13) with titers higher than those of $\operatorname{IgG}$ throughout $(11,14)$. Additionally, in some patients for whom leptospirosis is confirmed by culture and MAT, IgG never develops $(11,13)$.

We chose a commercially available IgM ELISA that has performed comparably to others in detecting serovars likely present in southern Sri Lanka (9). IgM is inherently cross-reactive, and thus serovars themselves are not detected. In a recent study from central Sri Lanka, the predominant serovars were Mednensis and Hardjo, but others included Australis, Ballum, Canicola, Celledoni, Cynopteri, Pomona, and Robinsoni (15). Previously, identification of the serovar Icterhemorrhagiae in Sri Lanka led to control of the rodent vector (16). The assay we used has reliably reacted with serovars Icterohemorrhagiae, Canicola, Grippotyphosa, Bataviae, Pomona, Tarassovi, Copenhageni, Bratislava, Hebdomadis, Sejroe, Australis, Panama, Pyrogenes, Patoc, Hardjo, and Cynopteri $(7,17)$.

Notably, detection of acute-phase IgM did not predict which patients had acute leptospirosis, despite its widespread use as an acute diagnostic test. Retrospective studies suggest sensitivities and specificities of 36\%-53\% for single acute-phase IgM and $90 \%-99 \%$ for MAT on paired serum specimens, respectively $(9,10)$. The varied sensitivity likely reflects different case definitions and control groups, timing of acute-phase specimen collection (up to 42 days after onset), geography and serovar distribution, platforms and protocols (e.g., ELISA \pm use of RF absorbent, indirect hemagglutination, and dot-ELISA and IgM dipsticks), and convenience sampling. Notably, ELISA of single (acutephase) serum specimens has performed as well or better than MAT or indirect hemagglutination of single serum specimens, so those strategies are not advised (10).

The most widely recognized problem with using acute-phase IgM to identify acute leptospirosis is that 
many persons in disease-endemic areas are expected to have preexisting antibodies. Some have advocated higher cut-offs to discriminate between acute infection and preexisting antibodies (1), because patients may be harmed as much by incorrectly attributing fever to leptospirosis as by falsely excluding it. However, data to support this approach are lacking, and misclassification could occur both early in acute infection (impaired sensitivity because antibody is not yet present) and later (impaired specificity because antibody is persistent). In our rigorous comparison of single vs. paired serum specimens, we found acute-phase IgM had especially poor sensitivity (17.5\%), since patients sought treatment early ( $\approx 3$ days), and more acute infections were identified by seroconversion than by a definitive rise in titer. The median duration of illness in those diagnosed by rise in titer versus seroconversion tended to be longer (4 days [IQR 3-5 days] vs. 3 days [IQR $2-5$ days], respectively; $\mathrm{p}=0.09$ ). Requiring a higher cutoff titer would further impair sensitivity. Hence, acute-phase IgM testing alone has multiple limitations for diagnosis of acute leptospirosis, regardless of the cut-off.

Only a few studies have evaluated the use of serologic testing for identifying leptospirosis in febrile cohorts. In Laos, 372 febrile patients were evaluated with ELISA (Panbio Ltd., Brisbane, Queensland, Australia) and immunochromatographic testing (ICT), which was compared with the MAT; acute leptospirosis (single titer $>400$ or 4 -fold rise in titer) was identified in $23(12.4 \%)$ of 186 patients (18). The sensitivity of ELISA and ICT was relatively high $(60.9 \%$ and $47.3 \%$, respectively), which could be explained by a long duration of fever (median 9 days). The sensitivity of ELISA for acute-phase versus convalescent-phase serum specimens was comparable (60.9\% and $65.2 \%$, respectively), but convalescent-phase serum specimens were obtained 4.5 days after acute-phase serum specimens. The specificity of both assays was similarly poor $(65.6 \%$ for ELISA and $75.5 \%$ for ICT). In Thailand, Cohen et al. identified acute leptospirosis in 67 (9.5\%) of febrile subjects using 2 rapid assays, a dipstick, and latex slide agglutination test (19). Patients sought treatment after a mean of 3.4 days of fever and returned 22 days later. Compared with MAT on paired serum specimens, the sensitivity of testing acute-phase serum with the dipstick and latex slide agglutination tests was $22 \%$ and $13 \%$, respectively, which is similar to our findings.

Strengths of our study include the rigorous, prospective design, uniquely large sample size, inclusion of an unstudied population believed to be at high risk, an unusually high rate of follow-up, and clinical correlation. To minimize selection bias, we used standardized criteria to sequentially enroll a large cohort ( $\approx 900$ patients) with thorough followup to enable assessment of acute-phase IgM testing versus clinical impression and relevant epidemiologic and clinical features. Those patients from whom paired serum specimens were not available differed only slightly from the included population. We excluded the few with equivocal results to avoid possible misclassification with resultant potential failure to identify significant predictive clinical features. By rigorously distinguishing acute from recent leptospirosis, we were able to confirm that myalgias and arthralgia were frequent symptoms and that the presence of conjunctivitis or conjunctival suffusion is diagnostically helpful. Leptospirosis in this cohort was relatively mild, as evidenced by stable vital signs, the absence of jaundice, and complete blood counts within nearly normal ranges. The sparse laboratory data reflect standard clinical practice in which automated testing is largely unavailable in the public sector, expensive in the private sector, and thus infrequently obtained.

Our results might have differed if we had used a different diagnostic standard, but culture, for example, is insensitive, labor intensive, unlikely to be available soon at Karapitya Teaching Hospital and many similar hospitals, and too slow to guide clinical management. We may have misclassified the number of days with fever, since temperatures are infrequently taken at home or in the hospital; however, duration of fever correlated well with that of symptoms, and no systematic bias would be expected, since etiologic diagnoses were not known when patients sought treatment at the hospital. Our estimate of leptospirosis may be low if a wider array of serovars is circulating in southern Sri Lanka than were detected by the ELISA used; however, no other available commercial assay would have been expected to be more sensitive.

We conclude that leptospirosis causes substantive illness in southern Sri Lanka. Furthermore, we found that testing acute-phase serum specimens for IgM has multiple limitations for the diagnosis of acute leptospirosis, because a positive result more often denoted past infection than an acute infection, and results were negative early in infection. Clinical impression is comparatively better without added cost (20). Paired serum specimens can provide rigorous diagnosis, but patients and clinicians need rapid diagnosis to guide clinical management. A few antigen-based or nucleic acid-based rapid tests have been described, but prospective clinical validations are limited $(17,21-25)$. Rapid, pathogen-based tests for early diagnosis need to be developed.

\section{Acknowledgments}

We thank the members of the microbiology laboratory at the Medical Faculty, University of Ruhuna, and the clinical staff at Karapitiya Teaching Hospital for their assistance.

Patient enrollment was supported by the Hubert-Yeargan Center for Global Health and the Duke University Medical Center Chancellor's Tsunami Relief Fund. Laboratory testing and M.E.R. 
were supported by a Johns Hopkins Center for Global Health Junior Faculty Grant, a Clinician Scientist Career Development Award from Johns Hopkins School of Medicine, and the National Institute of Allergy and Infectious Dieseases, National Institutes of Health (K23AIO83931).

Dr Reller is a pediatric and adult infectious diseases physician, medical microbiologist, and clinical investigator. Her main research interests include study of the epidemiology of acute febrile illness and its improved diagnosis.

\section{References}

1. Levett PN. Leptospirosis. Clin Microbiol Rev. 2001;14:296-326. doi:10.1128/CMR.14.2.296-326.2001

2. Sehgal SC. Epidemiological patterns of leptospirosis. Indian J Med Microbiol. 2006;24:310-1. doi:10.4103/0255-0857.29405

3. Pappas G, Papadimitriou P, Siozopoulou V, Christou L, Akritidis N. The globalization of leptospirosis: worldwide incidence trends. Int J Infect Dis. 2008;12:351-7. doi:10.1016/j.ijid.2007.09.011

4. Colombo Ministry of Health Epidemiology Unit. Surveillance of leptospirosis. Weekly Epidemiol Report Epi Unit. 2007;34:1-3.

5. Victoriano AF, Smythe LD, Gloriani-Barzaga N, Cavinta LL, Kasai T, Limpakarnjanarat K, et al. Leptospirosis in the Asia Pacific region. BMC Infect Dis. 2009;9:147. doi:10.1186/1471-2334-9-147

6. Agampodi S, Peacock SJ, Thevanesam V. The potential emergence of leptospirosis in Sri Lanka. Lancet Infect Dis. 2009;9:524-6. doi:10.1016/S1473-3099(09)70211-7

7. Virion/Serion. Serion ELISA classic Leptospira IgG/IgM (quantitatitive) instructions [cited $2010 \mathrm{Apr} 28$ ]. http://www.virion-serion. de

8. Cumberland P, Everard CO, Levett PN. Assessment of the efficacy of an IgM-Elisa and microscopic agglutination test (MAT) in the diagnosis of acute leptospirosis. Am J Trop Med Hyg. 1999;61:731-4.

9. Effler PV, Bogard AK, Domen HY, Katz AR, Higa HY, Sasaki DM. Evaluation of eight rapid screening tests for acute leptospirosis in Hawaii. J Clin Microbiol. 2002;40:1464-9. doi:10.1128/ JCM.40.4.1464-1469.2002

10. Bajani MD, Ashford DA, Bragg SL, Woods CW, Aye T, Spiegel RA, et al. Evaluation of four commercially available rapid serologic tests for diagnosis of leptospirosis. J Clin Microbiol. 2003;41:803-9. doi:10.1128/JCM.41.2.803-809.2003

11. Adler B, Murphy AM, Locarnini SA, Faine S. Detection of specific anti-leptospiral immunoglobulins $M$ and $G$ in human serum by solid-phase enzyme-linked immunosorbent assay. J Clin Microbiol. 1980;11:452-7.

12. Silva MV, Camargo ED, Batista L, Vaz AJ, Brandao AP, Nakamura $\mathrm{PM}$, et al. Behaviour of specific IgM, IgG and IgA class antibodies in human leptospirosis during the acute-phase of the disease and during convalescence. J Trop Med Hyg. 1995;98:268-72.

13. Adler B, Faine S. The antibodies involved in the human immune response to leptospiral infection. J Med Microbiol. 1978;11:387-400. doi:10.1099/00222615-11-4-387
14. Chernukha YG, Shishkina ZS, Baryshev PM, Kokovin IL. The dynamics of IgM- and IgG-antibodies in leptospiral infection in man. Zentralbl Bakteriol [Orig A]. 1976;236:336-43.

15. Agampodi SB, Thevanesam V, Wimalarathna H, Senarathna T, Wijedasa MH. A preliminary study on prevalent serovars of leptospirosis among patients admitted to teaching hospital, Kandy, Sri Lanka. Indian J Med Microbiol. 2008;26:405-6. doi:10.4103/02550857.43557

16. Babudieri B, Jagels G. Serological research on the presence of leptospirosis in Ceylon. Ceylon Med J. 1962;7:213-4.

17. Trombert-Paolantoni S, Thomas P, Hermet F, Clairet V, Litou N, Maury L. Dépistage de la Leptospirose: performance de la trousse Sérion Elisa classic Leptospira IgM ${ }^{\circledR}$ Kit. Pathol Biol (Paris). 2010;58:95-9. doi:10.1016/j.patbio.2009.06.008

18. Blacksell SD, Smythe L, Phetsouvanh R, Dohnt M, Hartskeerl R, Symonds M, et al. Limited diagnostic capacities of two commercial assays for the detection of Leptospira immunoglobulin M antibodies in Laos. Clin Vaccine Immunol. 2006;13:1166-9. doi:10.1128/ CVI.00219-06

19. Cohen AL, Dowell SF, Nisalak A, Mammen MP Jr, Petkanchanapong W, Fisk TL. Rapid diagnostic tests for dengue and leptospirosis: antibody detection is insensitive at presentation. Trop Med Int Health. 2007;12:47-51. doi:10.1111/j.1365-3156.2006.01752.x

20. Suputtamongkol Y, Pongtavornpinyo W, Lubell Y, Suttinont C, Hoontrakul S, Phimda K, et al. Strategies for diagnosis and treatment of suspected leptospirosis: a cost-benefit analysis. PLoS Negl Trop Dis. 2010;4:e610. doi:10.1371/journal.pntd.0000610

21. Djadid ND, Ganji ZF, Gouya MM, Rezvani M, Zakeri S. A simple and rapid nested polymerase chain reaction-restriction fragment length polymorphism technique for differentiation of pathogenic and nonpathogenic Leptospira spp. Diagn Microbiol Infect Dis. 2009;63:251-6. doi:10.1016/j.diagmicrobio.2008.10.017

22. Ooteman MC, Vago AR, Koury MC. Evaluation of MAT, IgM ELISA and PCR methods for the diagnosis of human leptospirosis. J Microbiol Methods. 2006;65:247-57. doi:10.1016/j.mimet.2005.07.015

23. Slack A, Symonds M, Dohnt M, Harris C, Brookes D, Smythe L. Evaluation of a modified Taqman assay detecting pathogenic Leptospira spp. against culture and Leptospira-specific IgM enzymelinked immunosorbent assay in a clinical environment. Diagn Microbiol Infect Dis. 2007;57:361-6. doi:10.1016/j.diagmicrobio.2006.10.004

24. Stoddard RA, Gee JE, Wilkins PP, McCaustland K, Hoffmaster AR. Detection of pathogenic Leptospira spp. through TaqMan polymerase chain reaction targeting the LipL32 gene. Diagn Microbiol Infect Dis. 2009;64:247-55. doi:10.1016/j.diagmicrobio. 2009.03.014

25. Ahmed A, Engelberts MF, Boer KR, Ahmed N, Hartskeerl RA. Development and validation of a real-time PCR for detection of pathogenic Leptospira species in clinical materials. PLoS ONE. 2009;4:e7093. doi:10.1371/journal.pone.0007093

Address for correspondence: Megan E. Reller, Johns Hopkins University School of Medicine, 720 Rutland Ave, Ross 624, Baltimore, MD 21205, USA; email: mreller1@jhmi.edu

\section{Medscape CME \\ Sign up to receive email announcements when a new article is available.}

\section{Get an online subscription at www.cdc.gov/ncidod/eid/subscrib.htm}

